\title{
Altered low-frequency oscillation amplitude of resting state-fMRI in patients with discogenic low-back and leg pain
}

This article was published in the following Dove Press journal: Journal of Pain Research

\author{
Fuqing Zhou ${ }^{1,2}$ \\ Lili $\mathrm{Gu}^{3}$ \\ Shunda Hong ${ }^{1,2}$ \\ Jiaqi Liu ${ }^{1,2}$ \\ Jian Jiang ${ }^{1,2}$ \\ Muhua Huang ${ }^{1,2}$ \\ Yong Zhang ${ }^{3}$ \\ Honghan Gong ${ }^{1,2}$ \\ 'Department of Radiology, The \\ First Affiliated Hospital, Nanchang \\ University, Nanchang, Jiangxi, People's \\ Republic of China; ${ }^{2}$ Neuroradiology \\ Laboratory, Jiangxi Province Medical \\ Imaging Research Institute, Nanchang, \\ Jiangxi, People's Republic of China; \\ ${ }^{3}$ Department of Pain Clinic, The \\ First Affiliated Hospital, Nanchang \\ University, Nanchang, Jiangxi, People's \\ Republic of China
}

Objective: The aim of this study was to explore the amplitude of intrinsic low-frequency oscillations (LFOs) in patients with discogenic low-back and leg pain (LBLP).

Participants and methods: We obtained and compared the LFO amplitude from 25 righthanded discogenic LBLP patients (13 males; mean age 55.16 \pm 1.83 years) and 27 well-matched healthy controls ( 15 males; mean age $52.96 \pm 1.63$ years). The LFO amplitude was examined using the voxel-wise amplitude of low-frequency fluctuations (ALFFs), and partial correlation analysis was performed to determine the relationship between the regions with altered ALFF values and clinical parameters in discogenic LBLP patients.

Results: Compared with healthy controls, the patients with discogenic LBLP showed a significant increase in ALFF in the affective system of the pain matrix (left anterior cingulate cortex, right anterior insula/frontal operculum, and bilateral orbitofrontal cortex) and informationprocessing regions (middle occipital/temporal gyrus). In addition, a significant decrease in ALFF was observed in the default mode network (DMN; inferior parietal lobule (IPL) and medial prefrontal cortex [mPFC]) and the processing system of the pain matrix (basal ganglia/ thalamus/midbrain, postcentral gyrus [PoCG], and fusiform gyrus). Several regions with altered ALFF were associated with disease duration, visual analog scale scores, Barthel index, and fine sensory modality measurements (two-point tactile discrimination of the left and/or right leg). Further operating characteristic curves analysis suggested that the mean ALFF values in the right IPL, left IPL/PoCG, left anterior cingulate cortex, and left mPFC could serve as markers to separate individuals with discogenic LBLP from healthy subjects.

Conclusion: Our results revealed widespread abnormalities in ALFF in the pain matrix and information-processing regions as well as a decrease in ALFF in the DMN. These results open up an important new avenue to better understand the nature of the link between intrinsic activity and peripheral pain and sensory impairment in discogenic LBLP patients.

Keywords: amplitude of low-frequency fluctuations, resting state functional magnetic resonance imaging, discogenic low-back and leg pain, amplitude of low-frequency fluctuations

\section{Introduction}

Low-back pain is a common health condition and results in large economic and social costs. ${ }^{1}$ For low-back pain, the common presentation is acute or chronic pain in the back and leg that can vary from a dull constant ache to a sudden sharp sensation. ${ }^{1}$ There is increasing concern that low-back pain can affect cerebral function and cause central impairment beyond the sensation of pain, including cortical plasticity. Functional restoration due to pain may influence clinical symptoms, manifestations, and rehabilitation..$^{2-4}$
Yong Zhang

Department of Pain Clinic, The First Affiliated Hospital, Nanchang University, 17 Yongwaizheng Street, Nanchang 330006, Jiangxi Province, People's Republic of China

Tel +8679188692643

Email zy830226@।63.com 
Structural and functional alterations related to low-back pain or chronic back pain have been demonstrated using advanced neuroimaging techniques. Examples of these alterations include neuron metabolite abnormities; ${ }^{5}$ cortical atrophy in the so-called "pain matrix", including the S1/S2 regions, insula, and medial prefrontal cortex (mPFC); ${ }^{6,7}$ cortical thinning in the "pain matrix" and pain-processing regions (trending in S1), ${ }^{8}$ white matter microstructure damage in the corpus callosum and internal capsule $;^{9-11}$ hyper-activation in the $\mathrm{mPFC}$ after thermal stimulation ${ }^{11}$ and activity in painassociated regions (thalamus and cingulate cortex) following mechanical stimulation; ${ }^{12}$ and highly consistent functional connectivity reorganization in several regions ${ }^{11,13-17}$ with the exception of disrupted default mode network (DMN) connectivity. ${ }^{14-16,18}$

Discogenic low-back pain can be caused by herniated disks, compressed nerve roots, and degenerative disks. In these patients, pain that radiates down the leg below the knee, with or without numbness, and is located on one side or both sides is called discogenic low-back and leg pain (LBLP). However, the nature of the intrinsic neuronal activity of discogenic LBLP has not been fully investigated and the relationship between cerebral function and clinical associations is not fully understood.

Resting-state functional magnetic resonance imaging (rs-fMRI) is an effective method to investigate the intrinsic neuronal activity in various neuropsychiatric and neuropsychological diseases, including Alzheimer's disease, ${ }^{19}$ mild cognitive impairment, ${ }^{20}$ multiple sclerosis,${ }^{21}$ neuromyelitis optica, ${ }^{22}$ posttraumatic stress disorder, ${ }^{23}$ and schizophrenia. ${ }^{24}$ In rs-fMRI, low-frequency $(0.01-0.08 \mathrm{~Hz})$ spontaneous blood oxygen level-dependent (BOLD) fluctuations are physiologically meaningful and are related to neural spontaneous activity. The amplitude of low-frequency fluctuations (ALFFs) is a reliable and reproducible method that focuses on regions of spontaneous activity at the voxel level. ALFF is calculated to measure the amplitude values of cortical low-frequency oscillations (LFOs) of regional rs-fMRI time courses. ${ }^{25,26}$

In this study, our hypothesis was that discogenic LBLP would alter the amplitude of oscillations of local neural activity, which in turn would be related to clinical status and may provide additional information about brain dysfunction. In the present study, we applied ALFF to examine the amplitude of LFOs in discogenic LBLP and healthy groups and then compared the results across patients and controls. To assess clinical relevance, we investigated the alteration of baseline brain activity and correlated it with disease severity, duration, visual analog scale (VAS) scores, and the severity of damage to fine sensory modalities.

\section{Participants and methods}

This case-control study was approved by the Medical Research Ethics Committee and the Institutional Review Board of The First Affiliated Hospital, Nanchang University, People's Republic of China. Written informed consent was obtained from all subjects, and the study was conducted according to the principles of the Declaration of Helsinki.

\section{Participants}

Thirty right-handed discogenic LBLP patients with lumbar disk herniation were recruited at The First Affiliated Hospital of Nanchang University from October 2016 to July 2017. All subjects underwent an evaluation that included computed tomography (CT) or magnetic resonance imaging (MRI) scanning and were diagnosed with more than one ruptured annulus fibrosus and compressed soft tissues. The resultant LBLP was ongoing for at least 1 month, and conservative medication, exercise, and physical therapy failed to improve the patients' condition.

The inclusion criteria were as follows: 1) age of 35-65 years; 2) clear evidence of compression of the spinal canal on a lumbar CT and/or MRI (Figure 1); 3) radiating pain (VAS score $>4$ ) from the lumbar region to the buttocks and lower limb; increased pain from abdominal pressure (cough and sneeze); 4) straight-leg raising test and pick-up test showing a positive reaction; knee and Achilles tendon reflex weakened or absent; 5) self-report of medication with nonsteroidal anti-inflammatory drugs (eg, Motrin, Advil, and Naproxen) and acetaminophen (eg, Tylenol) without the use of opioids; and 6) volunteered to enroll in the study.

The exclusion criteria were as follows: 1) previous spinal surgery or spinal abnormality; 2) trauma or infection

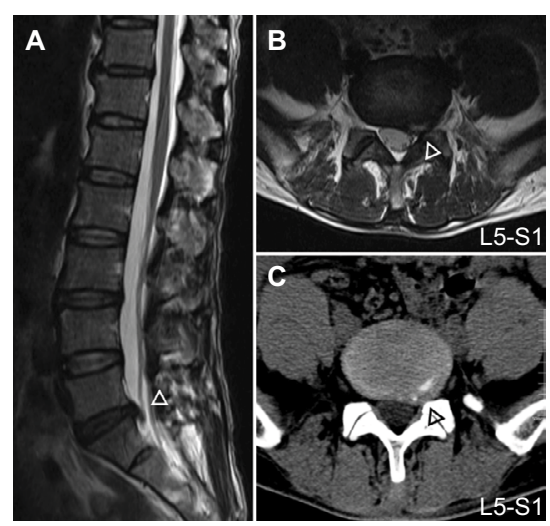

Figure I Sagittal (A), axial T 2 images (B) and axial CT images (C) of spinal canal and lateral recess $(\Delta)$ compression in discogenic LBLP and $H C$ subjects. Abbreviations: $\mathrm{CT}$, computed tomography; HC, healthy control; LBLP, low-back and leg pain. 
related to spinal canal compression; 3) other neurological disorders such as multiple sclerosis and a history of trauma; 4) cardio-cerebrovascular, liver, kidney, and hematopoietic system diseases; 5) presence of other calcification in disk protrusion, lateral recess stenosis, spinal stenosis, piriformis syndrome, or sciatica of the trunk; 6) diagnosed as having lumbar disk herniation without clinical symptoms; 7) refusal by the patient to enroll; and 8) MRI contraindications, such as metallic implants, claustrophobia or devices in the body, or subjects who did not finish the MRI scans or neuropsychological tests. For image quality control, participants with maximum displacements of rs-fMRI in the orthogonal directions $(x, y, z)$ of $>3 \mathrm{~mm}$ or a maximum rotation $(x, y, z)$ of $>3.0^{\circ}$ in $\max$ head motion were excluded.

\section{Clinical measures}

The authors evaluated patients using a VAS (0-10) score for pain rating, Japanese Orthopedic Association (JOA; -6 to 29) score for disease severity, ${ }^{27}$ and Barthel index (0-100) for performance in activities of daily living. Additionally, the two-point tactile discrimination test, which measures fine sensory abilities, was used to assess tactile spatial resolution. ${ }^{28}$ All the clinical measures were assessed before magnetic resonance scanning.

\section{Image acquisition}

All magnetic resonance data were acquired using a 3.0 Tesla Siemens whole-body scanner with an eight-channel radiofrequency head coil (Trio Tim; Siemens, Munich, Germany). The brain fMRI scan acquisition included 30 4-mm thick interleaved axial slices with an interslice gap of $1.2 \mathrm{~mm}$, repetition time $(\mathrm{TR}) /$ echo time $(\mathrm{TE})=2,000 / 30 \mathrm{~ms}$, matrix $=64 \times 64$, and field of view $(\mathrm{FOV})=220 \mathrm{~mm} \times 220 \mathrm{~mm}$. During the rs-fMRI scan, subjects were asked to keep their eyes closed and not to think about anything in particular and not to fall asleep for $8 \mathrm{~min}$ (240 time points). T1-weighted $\left(\mathrm{T}_{1} \mathrm{~W}\right)$ magnetization-prepared rapid gradient-echo structural images were acquired using the following parameters: TR/ $\mathrm{TE}=1,900 \mathrm{~ms} / 2.26 \mathrm{~ms} ; \mathrm{FOV}=215 \mathrm{~mm} \times 230 \mathrm{~mm}$; matrix $=240 \times 256$; and 176 1.0-mm thick sagittal slices with no gap. Additional conventional $\mathrm{T}_{2}$-weighted $\left(\mathrm{T}_{2} \mathrm{~W}\right)$ and $\mathrm{T}_{2}$-fluidattenuated inversion recovery images were acquired in the brain to diagnose each subject. For diagnosis in each subject, sagittal and axial conventional $\mathrm{T}_{1} \mathrm{~W}, \mathrm{~T}_{2} \mathrm{~W}$, and $\mathrm{T}_{2}$ fat-suppression sequences were acquired in the lumbar spine and disks from L1 to S3. At the end of the scanning sessions, all participants reported that they had not fallen asleep during the scan, according to the Epworth Sleepiness Scale questionnaire.

\section{rs-fMRI preprocessing and ALFF computing}

The preprocessing of rs-fMRI data was performed using the toolbox for Data Processing \& Analysis for Brain Imaging (DPABI v2.3, http://www.rfmri.org/dpabi) and included the following: the first 10 time points were discarded for stabilization; slice-timing correction; motion correction; spatial realignment; registration and normalization (in Montreal Neurological Institute [MNI] 152 space); resampling with $3 \mathrm{~mm}^{3}$ cubic voxels and spatial smoothing with $6 \mathrm{~mm}$ full-width-halfmaximum Gaussian kernel; and linear regression to reduce the contribution of nonneuronal fluctuations, including head motion parameters, white matter signals, cerebrospinal fluid signals, and global signals (to reduce motion effect ${ }^{29}$ ). For the motion correction, the following two steps were adopted to reduce the effects of head motion: 1) the three rotational and three translational motion parameters were computed for the exclusion of the hypermotion subjects (criteria: $3.0 \mathrm{~mm}$ and $3.0^{\circ}$ for max head motion); 2) the Friston 24-parameter model $^{30}$ was calculated for individual-level motion regression, including six head motion parameters, six head motion parameters one time point before, and the 12 corresponding squared items. For precise spatial normalization of the fMRI data, high-resolution individual T1-weighted magnetization prepared rapid gradient echo structural images were registered to the mean fMRI data, and the resulting aligned T1-weighted images were segmented and transformed into standard MNI spaces using the Diffeomorphic Anatomical Registration Through Exponentiated Lie Algebra toolbox. ${ }^{31}$

The ALFF analysis was performed using the DPABI, ${ }^{32}$ which has been described previously. ${ }^{33}$ Briefly, the preprocessing time series was transformed to the frequency domain using the fast Fourier transform algorithm. Typically, temporal band-pass filtering $(0.01<f<0.1 \mathrm{~Hz})$ was then performed for each voxel to reduce the effects of low frequency drift and high-frequency physiological noise, and the averaged square root was chosen as the ALFF (Equation 2) in the filtering frequency band. For standardization, the ALFF of each voxel was $z$-transformed with Fisher's $r$-to- $z$ transformation for subsequent group comparisons.

$$
\begin{aligned}
& x(t)=\sum_{k=1}^{N}\left[a_{k} \cos \left(2 \pi f_{k} t\right)+b_{k} \sin \left(2 \pi f_{k} t\right)\right] \\
& A L F F=\sum_{k: \int_{k}(0.01,0.1)} \sqrt{\left[a_{k}^{2}(f)+b_{k}^{2}(f) / N\right]}
\end{aligned}
$$

where $x(t)$ is a time series. 


\section{Statistical analysis}

\section{Between-group ALFF analysis}

For group comparisons, demographic data and head motion were compared using the two-sample $t$-test and $\chi^{2}$ test via SPSS (release 13.0; SPSS Inc., Chicago, IL, USA). The two-sample $t$-test was used to compare the $z$-ALFF values in each voxel of the two groups (two-tailed, voxel-level $P<0.01$; Gaussian random field theory (GRF) correction, cluster-level $P<0.05)$. The significant cluster was labeled with the coordinate and $t$-values of the peak voxel.

\section{Correlation analysis}

To investigate the relationship between cerebral abnormalities and clinical parameters (JOA, VAS, and so on), we first calculated the average value of ALFF within the clusters and significant ALFF changes were obtained via the two-sample $t$-test. A partial correlation analysis was performed to determine the relationship between the average ALFF values and clinical parameters in the patient group; the effects of age, sex, intracranial volume, and mean framewise displacement were considered. The multiple correlations were corrected using the Bonferroni correction.

\section{ROC analysis and discriminant function}

Several regions exhibited significant alterations in ALFF in the patients; thus, the ALFF value has the potential to be used as a biomarker to separate the discogenic LBLP patients from healthy subjects. To test this possibility, mean ALFF values were extracted from the altered regions and receiver operating characteristic (ROC) curves analysis was conducted.

\section{Results}

\section{Demographic and clinical data profiling}

Table 1 shows the general demographic and clinical information of discogenic LBLP patients and healthy controls. The data of 25 patients and 27 healthy subjects were analyzed. The following patients were excluded from the analysis: two patients with vascular malformation or infarction and three patients and two healthy subjects due to head motion. There were no significant differences between the groups with respect to age $(P=0.727)$ or sex $(P=0.654)$. All discogenic LBLP patients presented with low-back pain and lumbar disk herniation; $14(56 \%)$ patients presented with left leg pain and numbness; 9 (36\%) patients presented with right leg pain and numbness; and $2(8 \%)$ patients presented with bilateral leg symptoms. In the patients, two-point tactile discrimination
Table I Demographic data and clinical measures scores for the discogenic LBLP group and healthy controls

\begin{tabular}{llll}
\hline Subject & $\begin{array}{l}\text { Discogenic } \\
\text { LBLP }\end{array}$ & $\begin{array}{l}\text { Healthy } \\
\text { controls }\end{array}$ & P-value \\
\hline Participants (n) & 25 & 27 & $\mathrm{n} / \mathrm{a}$ \\
Age & $55.16 \pm 1.83$ & $52.96 \pm \mathrm{I} .63$ & 0.727 \\
Sex (male/female) & $13 / 12$ & $15 / 12$ & $0.84 I^{*}$ \\
Handedness (right/left) & $25 / 0$ & $25 / 0$ & $\mathrm{n} / \mathrm{a}$ \\
Duration of symptoms & $37.08 \pm 10.23$ & $\mathrm{n} / \mathrm{a}$ & $\mathrm{n} / \mathrm{a}$ \\
(months) & & & \\
Barthel index & $86.80 \pm 2.67$ & $100 \pm 0.0$ & $<0.000 \mathrm{I}$ \\
VAS scores & $5.78 \pm 0.21$ & $0 \pm 0$ & $<0.000 \mathrm{I}$ \\
JOA scores & $13.72 \pm \mathrm{I} .13$ & $28.96 \pm 0.04$ & $<0.000 \mathrm{I}$ \\
$\quad$ Subjective symptom & $3.60 \pm 0.37$ & $8.96 \pm 0.05$ & $<0.000 \mathrm{I}$ \\
$\quad$ Physical sign & $4.20 \pm 0.29$ & $6 \pm 0$ & $<0.000 \mathrm{I}$ \\
$\quad$ Daily activities & $6.04 \pm 0.72$ & $14 \pm 0$ & $<0.000 \mathrm{I}$ \\
$\quad$ Bladder dysfunction & $-0.12 \pm 0.60$ & $0 \pm 0$ & 0.303 \\
Mean head motion (mm) & $0.434 \pm 0.004$ & $0.375 \pm 0.004$ & 0.322 \\
ICV (mm ${ }^{3}$ ) & $0.774 \pm 0.007$ & $0.782 \pm 0.004$ & 0.323 \\
\hline
\end{tabular}

Notes: * $\chi^{2}$ test. Data presented as number or mean \pm standard error.

Abbreviations: ICV, intracranial volume; JOA, Japanese Orthopaedic Association; LBLP, low-back and leg pain; n/a, not applicable; VAS, visual analog scale.

decreased to different degrees in the right $(30.8 \pm 1.65 \mathrm{~mm})$ and left $(31.1 \pm 1.21 \mathrm{~mm})$ feet and the right $(25.3 \pm 1.23 \mathrm{~mm})$ and left $(26.1 \pm 1.25 \mathrm{~mm})$ hands. There were significant differences in JOA and VAS scores between the discogenic LBLP group and control group. No significant differences were found in mean framewise displacement $(P=0.322)$ and intracranial volume $(P=0.323)$ between the two groups.

\section{ALFF pattern and alterations in discogenic LBLP patients}

Figure 2 shows the group mean ALFF values in discogenic LBLP patients and healthy controls. Comparatively, discogenic LBLP patients had significantly decreased mean ALFF values $(0.356 \pm 0.057$ vs $0.429 \pm 0.125, P=0.010)$. Table 2 and Figure 3 (red-yellow spots) show increased ALFF in the right cerebellar posterior lobe (CPL), right inferior temporal gyrus (ITG), left ITG, right anterior insula (aINS)/frontal operculum (fO), bilateral orbitofrontal cortex (OFC), right middle occipital gyrus (MOG)/middle temporal gyrus (MTG), left anterior cingulate cortex (ACC), and left MOG of LBLP patients when compared with healthy subjects. Significantly decreased ALFF was found in the right fusiform (Fus) gyrus, bilateral midbrain (MB)/basal ganglia (BG)/ thalamus (Thala), left mPFC, left inferior parietal lobule (IPL)/postcentral gyrus (PoCG) and right IPL (cyaneous blue spots in Figure 3). 


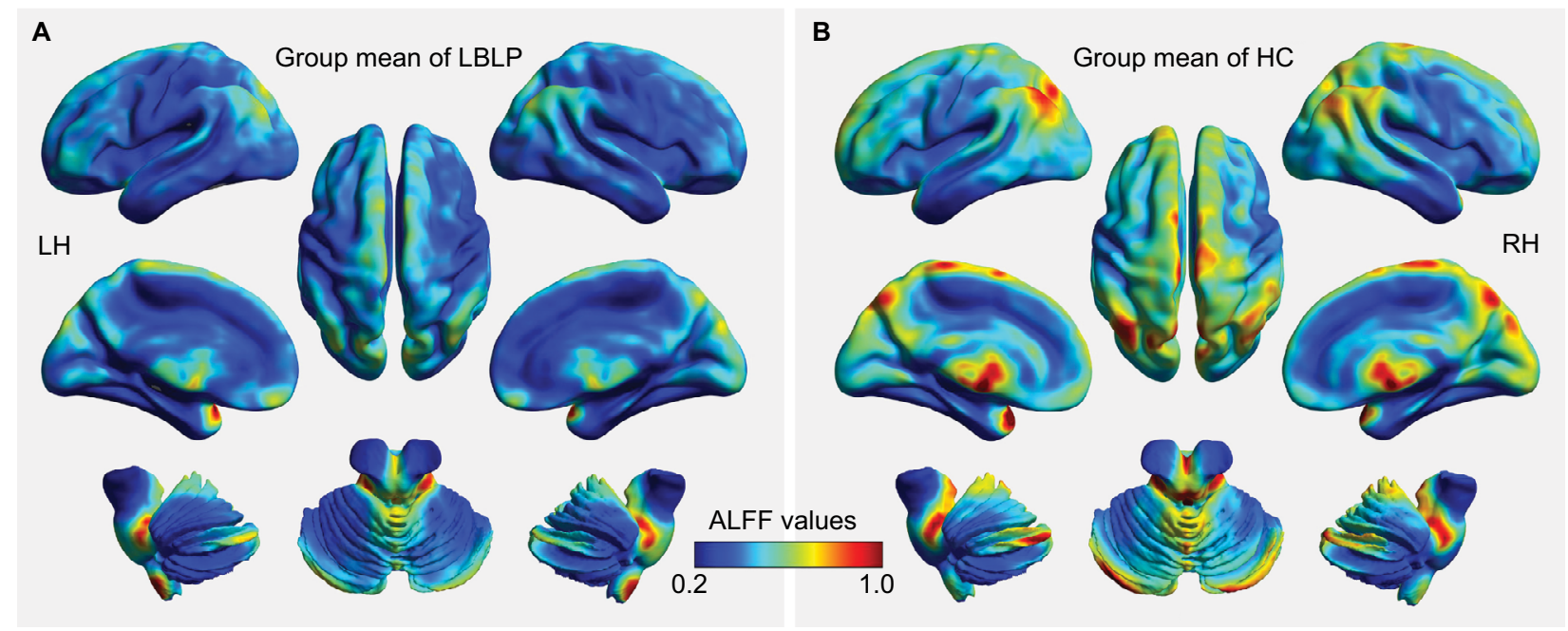

Figure 2 Whole-brain voxel-wise ALFF patterns in discogenic LBLP patients (A) and healthy subjects (B).

Abbreviations: ALFF, amplitude of low-frequency fluctuation; HC, healthy control; LBLP, low-back and leg pain; $\mathrm{LH}$, left hemisphere; RH, right hemisphere.

Table 2 The regions with altered ALFF in discogenic LBLP patients when compared with healthy subjects (two-tailed, voxel-level $P<0.0$ I; GRF correction, cluster-level $P<0.05$ )

\begin{tabular}{|c|c|c|c|c|c|c|}
\hline \multirow[t]{2}{*}{ Brain regions } & \multirow[t]{2}{*}{ BA } & \multirow{2}{*}{$\begin{array}{l}\text { Peak intensity } \\
\text { value }\end{array}$} & \multirow{2}{*}{$\begin{array}{l}\text { Number } \\
\text { of voxels }\end{array}$} & \multicolumn{3}{|c|}{ Peak location (MNI) } \\
\hline & & & & $x$ & $y$ & $\mathbf{z}$ \\
\hline \multicolumn{7}{|c|}{ LBLP patients $>$ healthy subjects } \\
\hline Right CPL & NA & 6.348 & 311 & 21 & -33 & -36 \\
\hline Right ITG & 20,21 & 7.868 & 158 & 57 & -12 & -27 \\
\hline Left ITG & 20,21 & 6.237 & 189 & -63 & -12 & -24 \\
\hline Right alNS/fO & $13,47,22$ & 5.764 & 188 & 51 & 21 & 0 \\
\hline Bilateral OFC & 11 & 4.794 & 102 & -3 & 66 & -15 \\
\hline Right MOG/MTG & 19,39 & 5.218 & 458 & 51 & -78 & 21 \\
\hline Left ACC & 24 & 7.086 & 236 & 12 & 21 & 12 \\
\hline Left MOG & 19 & 4.215 & 100 & -36 & -87 & 27 \\
\hline \multicolumn{7}{|c|}{ LBLP patients $<$ healthy subjects } \\
\hline Right Fus gyrus & $37,20,19$ & -4.591 & 140 & 51 & -54 & -21 \\
\hline Bilateral MB/BG/Thala & NA & -6.531 & 460 & 6 & 0 & -3 \\
\hline Left mPFC & 10 & -7.781 & 195 & -18 & 57 & 9 \\
\hline Left IPL/PoCG & 40,7 & -4.260 & 279 & -42 & -36 & 51 \\
\hline Right IPL & 40,7 & -5.673 & 160 & 36 & -57 & 48 \\
\hline
\end{tabular}

Abbreviations: ACC, anterior cingulate cortex; alNS, anterior insula; ALFF, amplitude of low-frequency fluctuation; BA, Brodmann area; BG, basal ganglia; CPL, cerebellar posterior lobe; fO, frontal operculum; Fus, fusiform; GRF, Gaussian random field theory; IPL, inferior parietal lobule; ITG, inferior temporal gyrus; LBLP, low-back and leg pain; MB, midbrain; MNI, Montreal Neurological Institute; MOG, middle occipital gyrus; mPFC, medial prefrontal cortex; MTG, middle temporal gyrus; NA, not applicable; OFC, orbitofrontal cortex; PoCG, postcentral gyrus; Thala, thalamus.

\section{Clinical associations of ALFF in discogenic LBLP patients}

Significant positive correlations between ALFF and disease duration were found in the left $\operatorname{mPFC}(P=0.022, \rho=0.472)$ and the right IPL $(P=0.049, \rho=0.414)$. The ALFF values in the right IPL correlated with VAS scores $(P=0.030, \rho=0.452)$ and the physical sign of the JOA index $(P=0.028, \rho=-0.458)$. The ALFF values of the right ITG correlated with the Barthel index $(P=0.039, \rho=-0.433)$ in the LBLP group (Figure 4 and
Table S1). Figure 5 and Table $\mathrm{S} 2$ show the correlation results between ALFF values and fine sensory modality measurements in the LBLP group. The ALFF values of the left MOG showed a significant negative correlation with the two-point tactile discrimination of the left foot $(P=0.004, \rho=-0.572)$ and right foot $(P=0.023, \rho=-0.472)$. ALFF values of the right ITG were significantly correlated with two-point tactile discrimination of the right hand $(P=0.038, \rho=0.438)$; ALFF values of the left IPL/PoCG were significantly correlated 


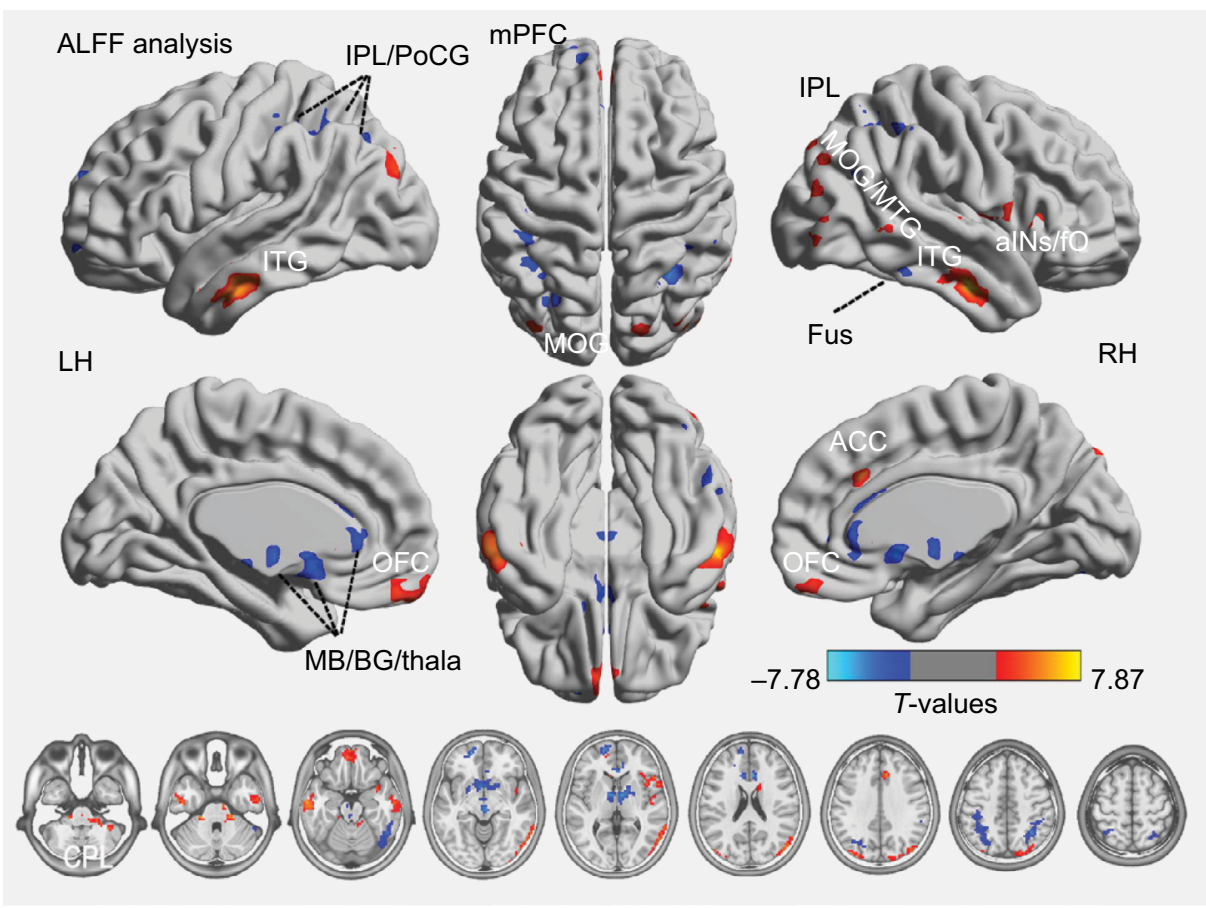

Figure 3 Differences in ALFF between discogenic LBLP and healthy subject groups (two-tailed, voxel-level $P<0.01$; GRF correction, cluster-level $P<0.05$ ) are shown. Notes: Warm colors indicate increases in ALFF, and cool colors indicate decreased ALFF in patients

Abbreviations: aINS, anterior insula; ALFF, amplitude of low-frequency fluctuation; BG, basal ganglia; CPL, cerebellar posterior lobe; fO, frontal operculum; Fus, fusiform; GRF, Gaussian random field theory; IPL, inferior parietal lobule; ITG, inferior temporal gyrus; LBLP, low-back and leg pain; LH, left hemisphere; MB, bilateral midbrain; MOG, middle occipital gyrus; mPFC, medial prefrontal cortex; MTG, middle temporal gyrus; OFC, orbitofrontal cortex; PoCG, postcentral gyrus; RH, right hemisphere; Thala, thalamus.

A

B

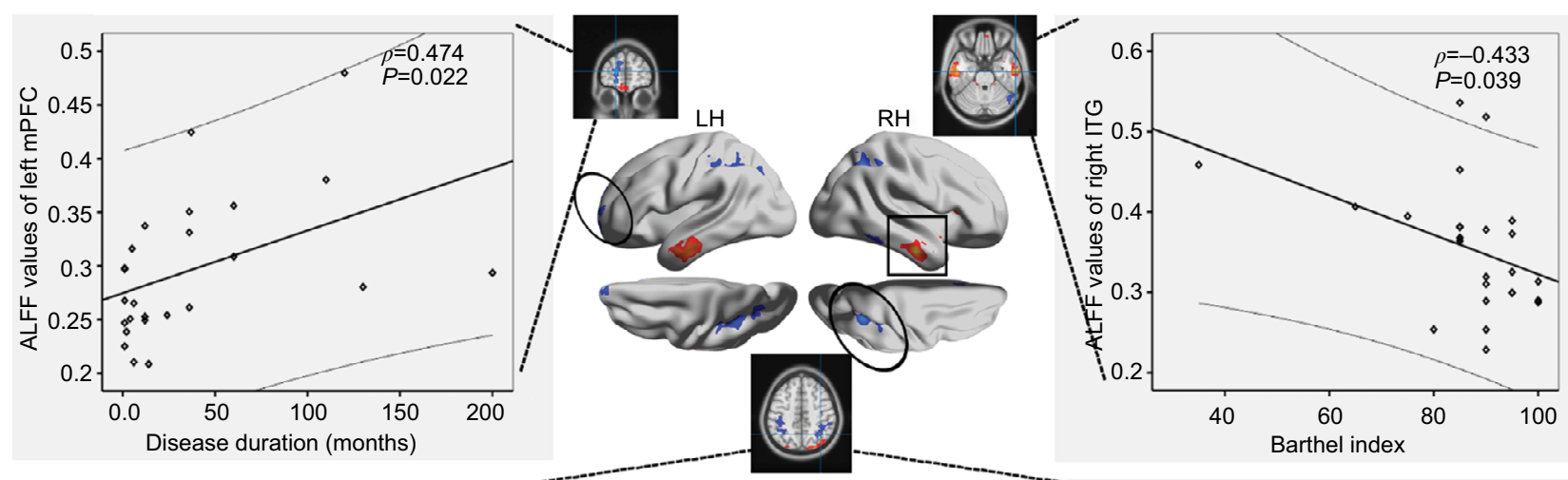

C
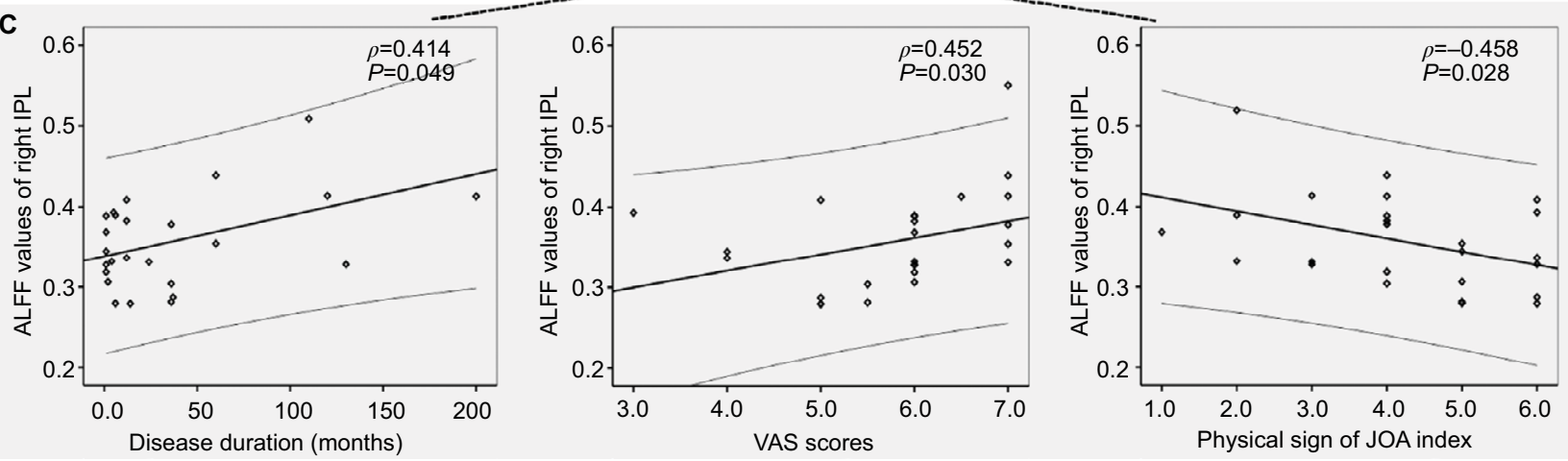

Figure 4 A significant correlation was observed between the ALFF values in the left mPFC (A), right ITG (B), and right IPL (C) and the clinical parameters (disease duration, VAS, and physical sign of JOA index) in the discogenic LBLP patient group.

Abbreviations: ALFF, amplitude of low-frequency fluctuation; IPL, infeior parietal lobule; ITG, inferior temporal gyrus; JOA, Japanese Orthopaedic Association; LBLP, lowback and leg pain; LH, left hemisphere; mPFC, medial prefrontal cortex; RH, right hemisphere; VAS, visual analog scale. 
A
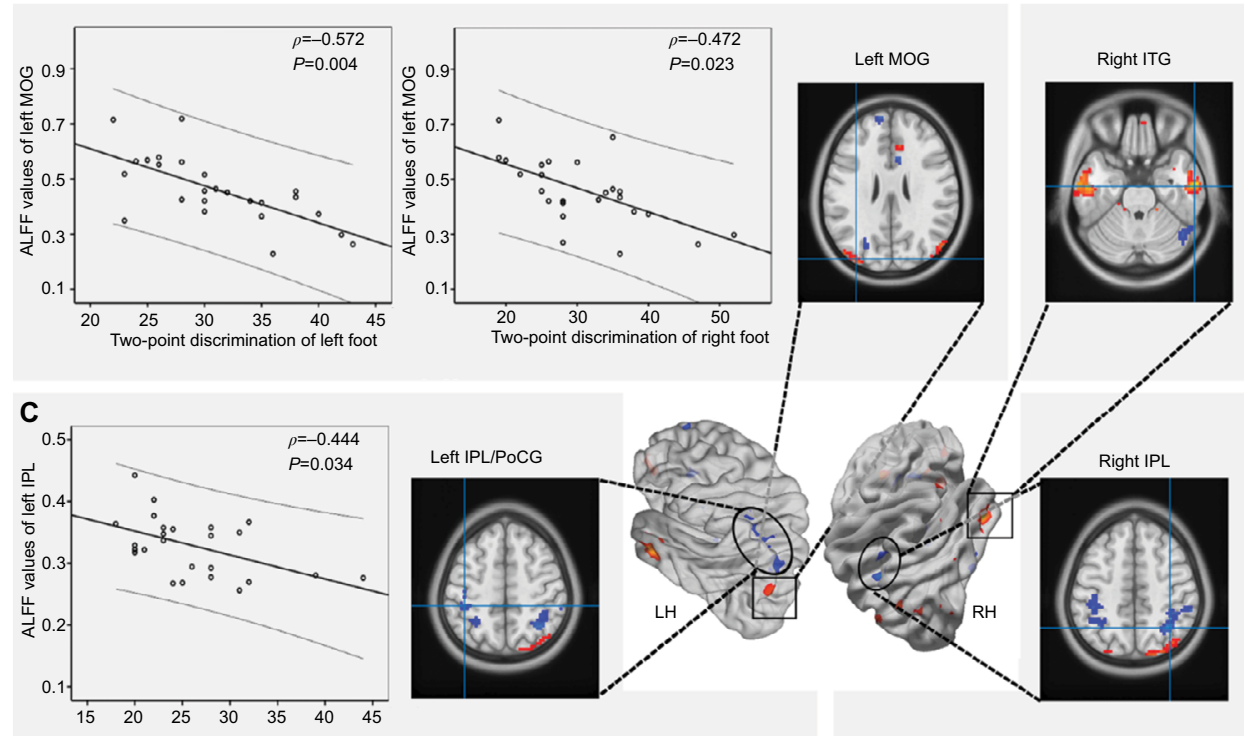

\section{B}
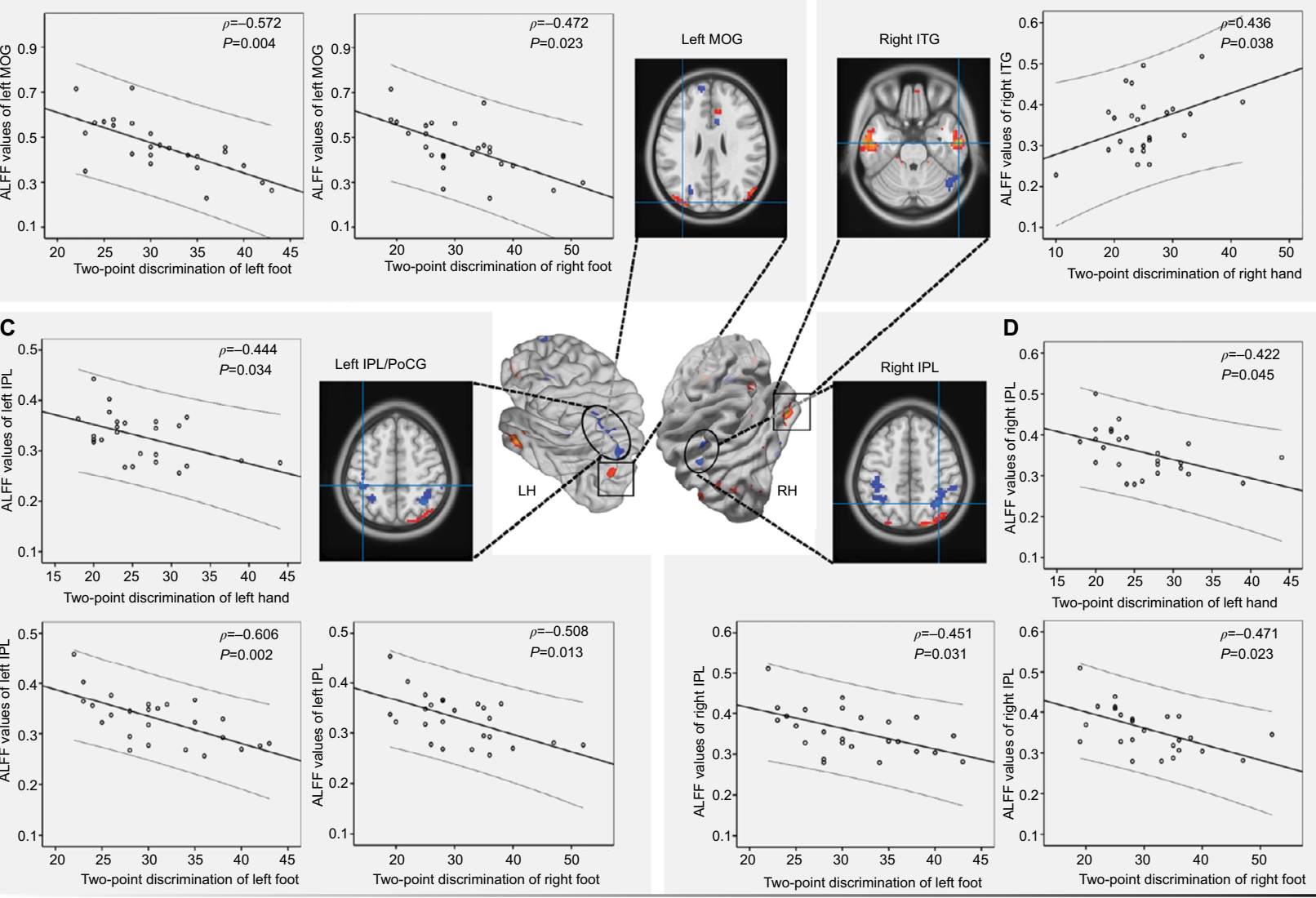

D
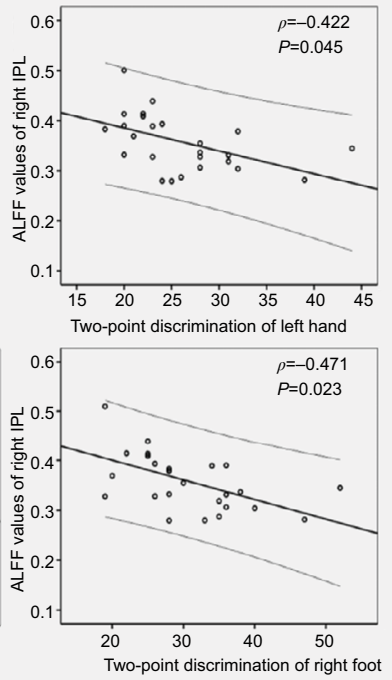

Figure 5 The correlation analysis results between the ALFF values of the left MOG (A), right ITG (B), left IPL/PoCG (C), and right IPL (D) and the fine sensory modality values in discogenic LBLP patients.

Abbreviations: ALFF, amplitude of low-frequency fluctuation; IPL, inferior parietal lobule; LBLP, low-back and leg pain; LH, left hemisphere; MOG, middle occipital gyrus; PoCG, postcentral gyrus; $\mathrm{RH}$, right hemisphere.

with the two-point tactile discrimination of the left hand ( $P=0.034, \rho=-0.444)$, left foot $(P=0.002, \rho=-0.505)$, and right foot $(P=0.013, \rho=-0.508)$. ALFF values of the right IPL were significantly correlated with the two-point tactile discrimination of the left hand $(P=0.045, \rho=-0.422)$, left foot $(P=0.031, \rho=-0.451)$, and right foot $(P=0.023, \rho=-0.471)$.

No significant correlation was found between the other brain indices and other clinical parameters (as shown in Tables S1 and S2).

\section{ROC analysis between the patient groups}

In some altered ALFF variables, discriminant functions showed excellent sensitivity and specificity (Figure 6 and Table 3). The area under the curve values of the right IPL (area under the curve $[\mathrm{AUC}]=0.883$ ), left IPL/PoCG (AUC $=0.904)$, left $\mathrm{ACC}(\mathrm{AUC}=0.692)$, left $\mathrm{mPFC}(\mathrm{AUC}=0.923)$, bilateral MB/BG/Thala (AUC $=0.929$ ), and right Fus (AUC $=0.902$ ) were relatively high, which demonstrated excellent classification accuracy for ALFF discriminant function.

\section{Discussion}

In this study, ALFF analysis was used to investigate the intrinsic LFO amplitude of discogenic LBLP patients and revealed abnormal neural activity in several cerebral regions. Compared with healthy controls, discogenic LBLP patients showed a significant increase in ALFF in the affective system of the pain matrix (left ACC, right aINS/fO, and bilateral OFC) and information-processing regions (MOG/ MTG and ITG) as well as a significant decrease in ALFF in the DMN (including IPL and $\mathrm{mPFC}$ ) and the processing system of the pain matrix (MB/BG/Thala, PoCG, and Fus). Several regions with altered ALFF were associated with disease duration, VAS scores, Barthel index, and fine sensory modality measurements (two-point tactile discrimination of the left and/or right leg). Further ROC analysis suggested that the mean ALFF values in the right IPL, left IPL/PoCG, left ACC, and left mPFC could serve as markers to separate individuals with discogenic LBLP from healthy subjects. 


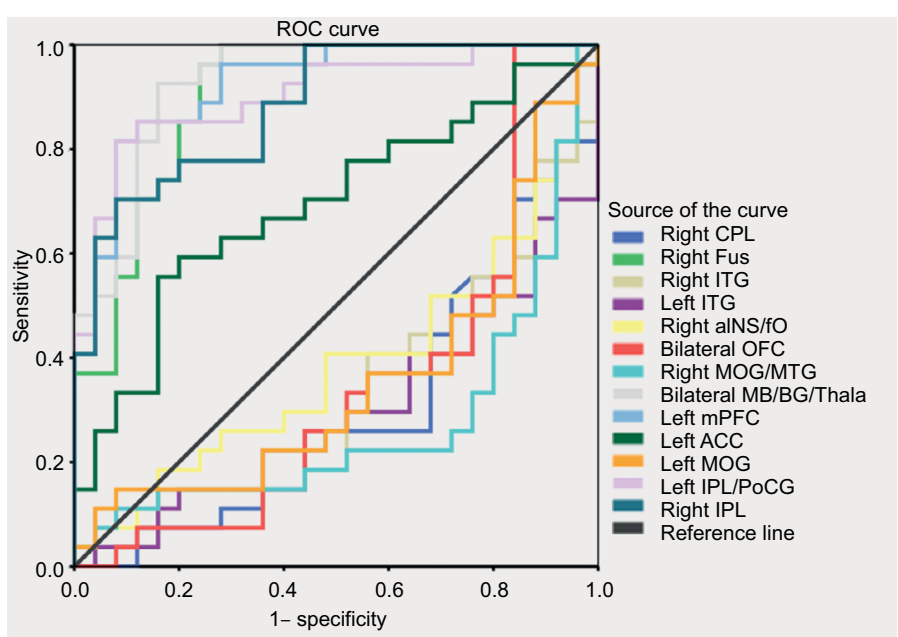

Figure 6 ROC analysis of ALFF in the altered regions as a potential mean to differentiate between discogenic LBLP patients and healthy subjects.

Abbreviations: ACC, anterior cingulate cortex; aINS, anterior insula; BG, basal ganglia; ALFF, amplitude of low-frequency fluctuation; CPL, cerebellar posterior lobe; fO, frontal operculum; Fus, fusiform; IPL, inferior parietal lobule; ITG, inferior temporal gyrus; LBLP, low-back and leg pain; MB, bilateral midbrain; MOG, middle occipital gyrus; mPFC, medial prefrontal cortex; MTG, middle temporal gyrus; OFC, orbitofrontal cortex; PoCG, postcentral gyrus; ROC, receiver operating characteristic; Thala, thalamus.

Table 3 ROC analysis for the differentiation between discogenic LBLP patients and healthy subjects

\begin{tabular}{lllll}
\hline Brain regions & Area under the curve & Cutoff point & Sensitivity (\%) & Specificity (\%) \\
\hline Right CPL & 0.300 & 0.305 & 56.0 & 16.0 \\
Right Fus & 0.902 & 0.368 & 96.0 & 7.41 \\
Right ITG & 0.329 & 0.351 & 24.0 & 48.15 \\
Left ITG & 0.299 & 0.289 & 40.0 & 25.92 \\
Right aINS/fO & 0.387 & 0.378 & 52.0 & 18.52 \\
Bilateral OFC & 0.345 & 0.492 & 40.0 & 25.92 \\
Right MOG/MTG & 0.271 & 0.318 & 28.0 & 25.92 \\
Bilateral MB/BG/Thala & 0.929 & 0.372 & 88.0 & 85.18 \\
Left mPFC & 0.923 & 0.366 & 84.0 & 92.59 \\
Left ACC & 0.692 & 0.296 & 60.0 & 81.48 \\
Left MOG & 0.354 & 0.376 & 40.0 & 29.6 \\
Left IPL/PoCG & 0.904 & 0.378 & 84.0 & 92.6 \\
Right IPL & 0.883 & $0.418^{\mathrm{a}}$ & 72.0 & 92.6 \\
\hline
\end{tabular}

Notes: With this cutoff point, the ALFF value of the right IPL could correctly classify 18 of the 25 discogenic LBLP patients and 25 of the 27 healthy subjects and resulted in a sensitivity of $72.0 \%$ and a specificity of $92.6 \%$. The means of other cutoff points were similar.

Abbreviations: ALFF, amplitude of low-frequency fluctuation; ACC, anterior cingulate cortex; aINS, anterior insula; BG, basal ganglia; CPL, cerebellar posterior lobe; fO, frontal operculum; Fus, fusiform; IPL, inferior parietal lobule; ITG, inferior temporal gyrus; LBLP, low-back and leg pain; MB, midbrain; MOG, middle occipital gyrus; mPFC, medial prefrontal cortex; MTG, middle temporal gyrus; OFC, orbitofrontal cortex; PoCG, postcentral gyrus; ROC, receiver operating characteristic; Thala, thalamus.

Back and leg pain evoked abnormal ALFF in the affective system of the pain matrix and sensory-processing regions. The pain matrix is composed of several interacting networks, including the posterior operculo-insular region (receiving spinothalamic projections), posterior parietal region, prefrontal and anterior insular areas (transition from a cortical nociceptive to a conscious state), and orbitofrontal and perigenual/limbic networks (for pain memories). Regarding the altered spatial pattern, the results of increased ALFF in the affective system of the pain matrix (left ACC, right aINS/fO, and bilateral OFC) were consistent with other functional neuroimaging studies. ${ }^{4,34}$ The aINS/fO involves multimodal sensory processing, sensory binding, and so on, while the ACC plays a role in a wide variety of autonomic functions. Considering the very low proportion of nociceptive-specific neurons in these brain areas, which is classically described in pain neuroimaging studies, the activation of these brain areas mainly reflects brain processes that are not directly related to the emergence of pain and that can be engaged by sensory inputs that do not originate from the activation of nociceptors. ${ }^{35}$ The OFC is a region known to be important in representing multiple levels of pain processing, influences bidding behavior and decision-making, ${ }^{36}$ and mediates pain inhibition. ${ }^{37}$ In patients with low-back pain or chronic pain, aINS/fO, ACC, and OFC activation (task fMRI) in response to unpleasant stimuli are largely comparable to those of healthy subjects with reduced gray 
matter, ${ }^{38}$ although there are some inconsistencies. ${ }^{39}$ The results of fMRI experiments ${ }^{38,40,41}$ suggest that attending to nociceptive pain leads to higher affective correlations or "side effects". Taken together, the present findings indicate that the increased ALFF in the affective system of the pain matrix supports previous findings concerning an automatic affective response to a putatively harmful stimulus.

Moreover, increased ALFF was detected in several information-processing regions, including the right MOG/ MTG, left MOG, and bilateral ITG in patients with discogenic LBLP. MOG showed a preference for spatial over nonspatial processing of both auditory and tactile stimuli and the modulation of unconscious processing. MTG, the exact function of which is unknown, has been connected with different processes such as contemplating distance. In patients with low-back pain, MOG/MTG might be activated by somatosensory stimuli. ${ }^{34,42}$ In the present study, increased ALFF in the left MOG was negatively correlated with fine sensory modality measurements (two-point tactile discrimination of the right and left feet), which indicates functional compensation or plasticity in partial sensory-processing regions. In healthy subjects, ITG is not only associated with visual stimuli processing but also involved in spatial awareness. Some processing of visual information corresponds to the visual processing ventral stream and is responsible for visual object recognition. In patients with LBLP, numbness is common in the leg due to intervertebral disk herniation that oppresses the nerve root. Decreased spatial information input from the lower extremity and/or regional poor efficiency might be the reason for increased ALFF in patients with discogenic LBLP. However, in the present study, a positive correlation was observed in the right ITG and fine sensory modality measurements (two-point tactile discrimination of the right hand). Combined with previous studies, our findings of increased ALFF point to a regional inefficiency in information-processing regions. This study found that there might be a connection between the poor efficiency of the right ITG and activities of daily living because a relationship has also been found between increased ALFF in the right ITG and the Barthel index.

Furthermore, we observed increased ALFF in the right $\mathrm{CPL}$, which plays an important role in fine motor coordination and has activity linked to happiness ${ }^{43}$ Previous imaging studies reported that low-back pain evoked increases in regional connectivity in the CPL in the resting state. ${ }^{44}$ The detailed implication of increased ALFF in the right CPL in discogenic LBLP patients is unclear, and further investigations are needed.
Another important finding is that the ALFF was decreased in the DMN (including the IPL and $\mathrm{mPFC}$ ) and the processing system of the pain matrix (MB/BG/Thala, PoCG, and Fus) in the patients with LBLP. In patients with chronic low-back pain, reduced deactivation was observed in the mPFC during a simple visual attention task $\mathrm{k}^{45}$ and disrupted restingstate connectivity in the mPFC ${ }^{18,46}$ and IPL was observed. ${ }^{47}$ Some studies have discussed functional connectivity during the whole period of fMRI data acquisition on the power spectrum. Therefore, the effects (increase/decrease) on the ALFF in discogenic LBLP could explain the alterations in brain region connectivity. Moreover, decreased ALFF in the left mPFC was associated with disease duration; thus, longterm injury to the low back by intervertebral disk herniation suggests that intrinsic activity was affected in the mPFC. Our results are consistent with previous studies involving chronic low-back pain. Combined with previous findings of cortical atrophy, ${ }^{6,7}$ a structural foundation for functional alteration in the DMN is provided.

Regarding the altered ALFF in the processing system of the pain matrix, additional observations supported the relationships between decreased ALFF in the right IPL and disease duration, VAS scores, or physical signs of the JOA index. In previous studies, similar atrophy and hypoactivity in sensory information transmission and processing regions were demonstrated in patients with chronic pain. ${ }^{6-8,12,45}$ In discogenic LBLP patients, injuries to the surrounding nerves might affect or prevent the function/movement of the related regions, causing a decrease in the gray matter in the corresponding somatosensory regions. In contrast to other nonspecific low-back pain, discogenic LBLP might be attributable to a recognizable specific pathology associated with central vulnerability and/or sensitivity to pain. ${ }^{48,49}$ In the present study, decreased ALFF in the right IPL or right IPL/ PoCG was negatively correlated with fine sensory modality measurements (two-point tactile discrimination of the left and/or leg), which is supported in individuals who are highly sensitive to pain and sensory impairment.

Compared to healthy controls, discogenic LBLP patients showed hyperamplitude of LFOs in the affective system of the pain matrix and information-processing regions and hypoamplitude of LFOs in the DMN and processing system of the pain matrix. There are reasons to believe that discogenic LBLP-related deficits in ALFF affect the capacity for neuroplasticity due to peripheral causes (nociceptive pain and sensory impairment), either adaptive or maladaptive; however, the exact underlying central mechanisms are still not fully understood. Partial ALFF differences between 
discogenic LBLP and HC groups might be useful diagnostic markers, and ALFF values in the right IPL, left IPL/PoCG, left ACC, left mPFC, bilateral MB/BG/Thala, and right Fus could help to accurately discriminate between these groups with a relatively high sensitivity and classification accuracy.

Some limitations exist in this study, although our results are interesting and encouraging. One limitation is that the present study was a case-control study, and we cannot completely rule out the effect of medication, although it is unlikely that the medication affected our results. It is important to note that oral morphine daily for 1 month could cause cerebral anatomical alterations; ${ }^{50}$ thus, we excluded all patients using opioids from this study. Another limitation is that this was an explorative study involving correlation analysis between the ALFF values and clinical fine sensory modality measurements, and we did not use other sensory measurements. Future studies are needed to address more comprehensive sensory assessments. For technical reasons, the head-motion models should be applied before the smoothing filters. After movement correction, any residual effect of motion artifacts in the fMRI can be decreased by a spatial low-pass filter. In this study, only ALFF, not fractional ALFF (fALFF), was used to probe the alterations in the discogenic LBLP patients. Although both ALFF and fALFF are sensitive and show moderate-to-high test-retest reliability in gray matter, the reliability of ALFF tends to be higher than that of fALFF. ${ }^{26}$ ALFF may be more sensitive to differences between groups and individuals because it is more reliable. Finally, the physiological basis of ALFF, the role of ALFF as a diagnostic marker, and recognizable patterns need to be examined in future work.

\section{Conclusion}

This study showed that altered ALFF in the pain matrix, $\mathrm{DMN}$, and information-processing regions was associated with clinical measurements in patients with discogenic LBLP. We believe that our results may provide evidence of an intrinsic plasticity link with peripheral causes (pain and sensory impairment) in discogenic LBLP patients.

\section{Acknowledgments}

This study was supported by the National Natural Science Foundation of China (81560284 and 81771808), the Jiangxi Province Science and Technology Support Program (20151BBG70224), the Natural Science Foundation of Jiangxi, People's Republic of China (grant number 20161BAB205245), and the Distinguished Young Scholars of Jiangxi Province (20171BCB23089).

\section{Disclosure}

The authors report no conflicts of interest in this work.

\section{References}

1. Zhang YG, Guo TM, Xiong G, Wu SX. Clinical diagnosis for discogenic low back pain. Int J Biol Sci. 2009;5(7):647-658.

2. Denk F, McMahon SB, Tracey I. Pain vulnerability: a neurobiological perspective. Nat Neurosci. 2014;17(2):192-200.

3. Butler S. Important new insight in pain and pain treatment induced changes in functional connectivity between the pain matrix and the salience, central executive, and sensorimotor networks. Scand J Pain. 2017; 16:64-65.

4. Chan A, Ford JJ, Surkitt LD, et al. Individualised functional restoration plus guideline-based advice vs advice alone for non-reducible discogenic low back pain: a randomised controlled trial. Physiotherapy. 2017;103(2):121-130.

5. Sharma NK, McCarson K, Van Dillen L, Lentz A, Khan T, Cirstea CM. Primary somatosensory cortex in chronic low back pain - a H-MRS study. J Pain Res. 2011;4:143-150.

6. Fritz HC, McAuley JH, Wittfeld K, et al. Chronic back pain is associated with decreased prefrontal and anterior insular gray matter: results from a population-based cohort study. J Pain. 2016;17(1):111-118.

7. Cauda F, Palermo S, Costa T, et al. Gray matter alterations in chronic pain: a network-oriented meta-analytic approach. Neuroimage Clin. 2014;4:676-686.

8. Dolman AJ, Loggia ML, Edwards RR, et al. Phenotype matters: the absence of a positive association between cortical thinning and chronic low back pain when controlling for salient clinical variables. Clin J Pain. 2014;30(10):839-845.

9. Ung H, Brown JE, Johnson KA, Younger J, Hush J, Mackey S. Multivariate classification of structural MRI data detects chronic low back pain. Cereb Cortex. 2014;24(4):1037-1044.

10. Čeko M, Shir Y, Ouellet JA, Ware MA, Stone LS, Seminowicz DA. Partial recovery of abnormal insula and dorsolateral prefrontal connectivity to cognitive networks in chronic low back pain after treatment. Hum Brain Mapp. 2015;36(6):2075-2092.

11. Buckalew N, Haut MW, Aizenstein H, et al. Differences in brain structure and function in older adults with self-reported disabling and nondisabling chronic low back pain. Pain Med. 2010;11(8):1183-1197.

12. Giesecke T, Gracely RH, Grant MA, et al. Evidence of augmented central pain processing in idiopathic chronic low back pain. Arthritis Rheum. 2004;50(2):613-623.

13. Baliki MN, Petre B, Torbey S, et al. Corticostriatal functional connectivity predicts transition to chronic back pain. Nat Neurosci. 2012;15(8):1117-1119.

14. Loggia ML, Kim J, Gollub RL, et al. Default mode network connectivity encodes clinical pain: an arterial spin labeling study. Pain. 2013;154(1):24-33.

15. Baliki MN, Baria AT, Apkarian AV. The cortical rhythms of chronic back pain. J Neurosci. 2011;31(39):13981-13900.

16. Baliki MN, Geha PY, Apkarian AV, Chialvo DR. Beyond feeling: chronic pain hurts the brain, disrupting the default-mode network dynamics. $J$ Neurosci. 2008;28(6):1398-1403.

17. Yang Q, Wang Z, Yang L, Xu Y, Chen LM. Cortical thickness and functional connectivity abnormality in chronic headache and low back pain patients. Hum Brain Mapp. 2017;38(4):1815-1832.

18. Tagliazucchi E, Balenzuela P, Fraiman D, Chialvo DR. Brain resting state is disrupted in chronic back pain patients. Neurosci Lett. 2010;485(1):26-31.

19. Dai Z, Yan C, Li K, et al. Identifying and mapping connectivity patterns of brain network hubs in Alzheimer's disease. Cereb Cortex. 2015;25(10):3723-3742.

20. Han Y, Wang J, Zhao Z, et al. Frequency-dependent changes in the amplitude of low-frequency fluctuations in amnestic mild cognitive impairment: a resting-state fMRI study. Neuroimage. 2011;55(1):287-295. 
21. Zhou F, Zhuang Y, Gong H, Zhan J, Grossman M, Wang Z. Resting state brain entropy alterations in relapsing remitting multiple sclerosis. PLoS One. 2016;11(1):e0146080.

22. Liu Y, Duan Y, Huang J, et al. Multimodal quantitative MR imaging of the thalamus in multiple sclerosis and neuromyelitis optica. Radiology. 2015;277(3):784-792.

23. Li S, Huang X, Li L, et al. Posttraumatic stress disorder: structural characterization with 3-T MR imaging. Radiology. 2016;280(2):537-544.

24. Tu PC, Hsieh JC, Li CT, Bai YM, Su TP. Cortico-striatal disconnection within the cingulo-opercular network in schizophrenia revealed by intrinsic functional connectivity analysis: a resting fMRI study. Neuroimage. 2012;59(1):238-247.

25. Zang YF, He Y, Zhu CZ, et al. Altered baseline brain activity in children with ADHD revealed by resting-state functional MRI. Brain Dev. 2007;29(2):83-91.

26. Zuo XN, Di Martino A, Kelly C, et al. The oscillating brain: complex and reliable. Neuroimage. 2010;49(2):1432-1445.

27. Yonenobu K, Abumi K, Nagata K, Taketomi E, Ueyama K. Interobserver and intraobserver reliability of the Japanese Orthopaedic Association scoring system for evaluation of cervical compression myelopathy. Spine. 2001;26(17):1890-1894.

28. Boldt R, Gogulski J, Gúzmanlopéz J, Carlson S, Pertovaara A. Twopoint tactile discrimination ability is influenced by temporal features of stimulation. Exp Brain Res. 2014;232(7):2179-2185.

29. Yan CG, Cheung B, Kelly C, et al. A comprehensive assessment of regional variation in the impact of head micromovements on functional connectomics. Neuroimage. 2013;76(8):183-201.

30. Friston KJ, Williams S, Howard R, Frackowiak RS, Turner R. Movement-related effects in fMRI time-series. Magn Reson Med. 1996;35(3): 346-355.

31. Goto M,Abe O, Aoki S, et al; Japanese Alzheimer's Disease Neuroimaging Initiative. Diffeomorphic anatomical registration through exponentiated lie algebra provides reduced effect of scanner for cortex volumetry with atlasbased method in healthy subjects. Neuroradiology. 2013;55(7):869-875.

32. Yan $\mathrm{CG}$, Wang XD, Zuo XN, Zang YF. DPABI: data processing $\&$ analysis for (resting-state) brain imaging. Neuroinformatics. 2016;14(3):339-351.

33. Yan CG, Zang YF. DPARSF: a MATLAB toolbox for "pipeline" data analysis of resting-state fMRI. Front Syst Neurosci. 2010;4:13.

34. Kregel J, Meeus M, Malfliet A, et al. Structural and functional brain abnormalities in chronic low back pain: a systematic review. Semin Arthritis Rheum. 2015;45(2):229-237.

35. Mouraux A, Diukova A, Lee MC, Wise RG, Iannetti GD. A multisensory investigation of the functional significance of the "pain matrix". Neuroimage. 2011;54(3):2237-2249.
36. Winston JS, Vlaev I, Seymour B, Chater N, Dolan RJ. Relative valuation of pain in human orbitofrontal cortex. J Neurosci. 2014;34(44): 14526-14535.

37. Becker S, Gandhi W, Pomares F, Wager TD, Schweinhardt P. Orbitofrontal cortex mediates pain inhibition by monetary reward. Soc Cogn Affect Neurosci. 2017;12(4):651-661.

38. Wasan AD, Loggia ML, Chen LQ, Napadow V, Jian K, Gollub RL. Neural correlates of chronic low back pain measured by arterial spin labeling. Anesthesiology. 2011;115(2):364-374.

39. Baliki MN, Schnitzer TJ, Bauer WR, Apkarian AV. Brain morphological signatures for chronic pain. PLoS One. 2011;6(10):e26010.

40. Lamm C, Nusbaum HC, Meltzoff AN, Decety J. What are you feeling? Using functional magnetic resonance imaging to assess the modulation of sensory and affective responses during empathy for pain. PLoS One. 2007;2(12):e1292.

41. Balagué F, Mannion AF, Pellisé F, Cedraschi C. Non-specific low back pain. Lancet. 2012;379(9814):482-491.

42. Pijnenburg M, Brumagne S, Caeyenberghs K, et al. Resting-state functional connectivity of the sensorimotor network in individuals with nonspecific low back pain and the association with the sit-to-stand-to-sit task. Brain Connect. 2015;5(5):303-311.

43. Schienle A, Scharmüller W. Cerebellar activity and connectivity during the experience of disgust and happiness. Neuroscience. 2013;246(9): 375-381.

44. Zhang SS, Wu W, Liu ZP, Huang GZ, Guo SG, Yang JM. Altered regional homogeneity in experimentally induced low back pain: a resting-state fMRI study. J Neuroeng Rehabil. 2014;11(1):115.

45. Baliki MN, Chialvo DR, Geha PY, et al. Chronic pain and the emotional brain: specific brain activity associated with spontaneous fluctuations of intensity of chronic back pain. J Neurosci. 2006;26(47): 12165-12173.

46. Letzen JE, Robinson ME. Negative mood influences default mode network functional connectivity in chronic low back pain patients: Implications for functional neuroimaging biomarkers. Pain. 2017;158(1): 48-57.

47. Zhang S, Wu W, Huang G, et al. Resting-state connectivity in the default mode network and insula during experimental low back pain. Neural Regen Res. 2014;9(2):135-142.

48. Taylor KS, Anastakis DJ, Davis KD. Cutting your nerve changes your brain. Brain. 2009;132(11):3122-3133.

49. Erpelding N, Moayedi M, Davis KD. Cortical thickness correlates of pain and temperature sensitivity. Pain. 2012;153(8):1602-1609.

50. Younger JW, Chu LF, D’Arcy NT, Trott KE, Jastrzab LE, Mackey SC. Prescription opioid analgesics rapidly change the human brain. Pain. 2011;152(8):1803-1810. 


\section{Supplementary materials}

Table SI Relationship between clinical status indices and the ALFF values in discogenic LBLP patients $(\rho$ values/P-values)

\begin{tabular}{|c|c|c|c|c|c|c|c|}
\hline & \multirow{2}{*}{$\begin{array}{l}\text { Disease } \\
\text { duration } \\
\text { (months) }\end{array}$} & \multirow[t]{2}{*}{ Barthel index } & \multirow[t]{2}{*}{ VAS scores } & \multicolumn{4}{|l|}{ JOA scores } \\
\hline & & & & $\begin{array}{l}\text { Total JOA } \\
\text { scores }\end{array}$ & $\begin{array}{l}\text { Subjective } \\
\text { Symptom }\end{array}$ & $\begin{array}{l}\text { Physical } \\
\text { sign }\end{array}$ & $\begin{array}{l}\text { Daily } \\
\text { activities }\end{array}$ \\
\hline Right CPL & $0.314 / 0.144$ & $-0.178 / 0.415$ & $0.382 / 0.072$ & $-0.128 / 0.560$ & $-0.158 / 0.47 \mid$ & $-0.3|6 / 0.14|$ & $0.098 / 0.657$ \\
\hline Right ITG/Fus & $0.059 / 0.265$ & $-0.011 / 0.960$ & $0.297 / 0.169$ & $0.013 / 0.952$ & $-0.033 / 0.882$ & $-0.26 \mathrm{I} / 0.228$ & $0.222 / 0.308$ \\
\hline Right ITG & $0.265 / 0.222$ & $-0.433 / 0.039 *$ & $0.252 / 0.246$ & $-0.155 / 0.48 \mid$ & $-0.106 / 0.631$ & $-0.335 / 0.119$ & $0.023 / 0.917$ \\
\hline Left ITG & $0.332 / 0.122$ & $-0.047 / 0.830$ & $0.164 / 0.455$ & $-0.001 / 0.997$ & $-0.051 / 0.818$ & $-0.091 / 0.679$ & $0.119 / 0.589$ \\
\hline Right alNS/fO & $0.179 / 0.414$ & $0.031 / 0.889$ & $0.192 / 0.380$ & $0.175 / 0.425$ & $0.139 / 0.528$ & $-0.275 / 0.204$ & $0.389 / 0.067$ \\
\hline Bilateral OFC & $0.254 / 0.24 I$ & $-0.060 / 0.786$ & $0.185 / 0.398$ & $-0.009 / 0.966$ & $0.084 / 0.704$ & $-0.35 \mathrm{I} / 0.100$ & $0.172 / 0.433$ \\
\hline Right MOG/MTG & $0.125 / 0.568$ & $-0.016 / 0.943$ & $0.024 / 0.912$ & $-0.016 / 0.943$ & $-0.146 / 0.506$ & $-0.013 / 0.952$ & $0.090 / 0.684$ \\
\hline Bilateral MB/BG/Thala & $0.136 / 0.537$ & $-0.291 / 0.178$ & $0.161 / 0.462$ & $0.017 / 0.937$ & $-0.071 / 0.747$ & $-0.189 / 0.389$ & $0.217 / 0.320$ \\
\hline Left mPFC & $0.474 / 0.022 *$ & $-0.054 / 0.808$ & $0.172 / 0.431$ & $-0.016 / 0.942$ & $-0.062 / 0.779$ & $-0.076 / 0.731$ & $0.084 / 0.703$ \\
\hline Left ACC & $0.208 / 0.342$ & $-0.306 / 0.155$ & $0.163 / 0.458$ & $0.182 / 0.407$ & $0.139 / 0.526$ & $-0.247 / 0.256$ & $0.400 / 0.058$ \\
\hline Left MOG & $0.124 / 0.572$ & $0.030 / 0.892$ & $0.119 / 0.588$ & $-0.029 / 0.896$ & $-0.135 / 0.539$ & $-0.110 / 0.618$ & $0.110 / 0.618$ \\
\hline Left IPL/PoCG & $0.211 / 0.355$ & $0.083 / 0.708$ & $0.341 / 0.112$ & $0.015 / 0.946$ & $0.099 / 0.651$ & $-0.323 / 0.132$ & $0.204 / 0.349$ \\
\hline Right IPL & $0.4 \mid 4 / 0.049 *$ & $-0.084 / 0.703$ & $0.452 / 0.030 *$ & $-0.157 / 0.474$ & $0.020 / 0.928$ & $-0.458 / 0.028 *$ & $0.034 / 0.879$ \\
\hline
\end{tabular}

Note: $* P<0.05$.

Abbreviations: ACC, anterior cingulate cortex; aINS, anterior insula; ALFF, amplitude of low-frequency fluctuation; BG, basal ganglia; CPL, cerebellar posterior lobe; fO, frontal operculum; Fus, fusiform; IPL, inferior parietal lobule; ITG, inferior temporal gyrus; JOA, Japanese Orthopaedic Association; LBLP, low-back and leg pain; MB, midbrain; MOG, middle occipital gyrus; mPFC, medial prefrontal cortex; MTG, middle temporal gyrus; OFC, orbitofrontal cortex; PFC, prefrontal cortex; PoCG, postcentral gyrus; Thala, thalamus; VAS, visual analog scale.

Table S2 Relationship between partial sensor measurements and the ALFF values in LBLP patients ( $\rho$ values/P-value)

\begin{tabular}{lllll}
\hline & TPTD of right hand & TPTD of left hand & TPTD of right foot & TPTD of left foot \\
\hline Right CPL & $-0.175 / 0.424$ & $-0.276 / 0.203$ & $-0.359 / 0.093$ & $-0.378 / 0.076$ \\
Right ITG/Fus & $-0.074 / 0.738$ & $-0.195 / 0.373$ & $-0.245 / 0.260$ & $-0.158 / 0.472$ \\
Right ITG & $0.436 / 0.038^{*}$ & $0.174 / 0.426$ & $0.099 / 0.654$ & $0.211 / 0.335$ \\
Left ITG & $0.128 / 0.561$ & $0.004 / 0.984$ & $-0.152 / 0.489$ & $-0.163 / 0.456$ \\
Right aINS/fO & $-0.016 / 0.943$ & $0.023 / 0.917$ & $-0.176 / 0.421$ & $-0.163 / 0.458$ \\
Bilateral OFC & $0.169 / 0.440$ & $0.113 / 0.607$ & $-0.031 / 0.888$ & $-0.150 / 0.493$ \\
Right MOG/MTG & $-0.291 / 0.177$ & $-0.292 / 0.176$ & $-0.260 / 0.230$ & $-0.378 / 0.076$ \\
Bilateral MB/BG/Thala & $-0.078 / 0.724$ & $-0.070 / 0.751$ & $-0.041 / 0.853$ & $-0.144 / 0.513$ \\
Left mPFC & $-0.104 / 0.638$ & $-0.115 / 0.601$ & $-0.293 / 0.176$ & $-0.397 / 0.061$ \\
Left ACC & $0.016 / 0.943$ & $-0.023 / 0.917$ & $-0.089 / 0.686$ & $-0.105 / 0.633$ \\
Left MOG & $-0.254 / 0.242$ & $-0.403 / 0.056$ & $-0.472 / 0.023 *$ & $-0.572 / 0.004^{*}$ \\
Left IPL/PoCG & $-0.394 / 0.063$ & $-0.444 / 0.034^{*}$ & $-0.508 / 0.013^{*}$ & $-0.606 / 0.002^{*}$ \\
Right IPL & $-0.244 / 0.261$ & $-0.422 / 0.045^{*}$ & $-0.471 / 0.023^{*}$ & $-0.451 / 0.03 I^{*}$ \\
\hline
\end{tabular}

Note: $* P<0.05$.

Abbreviations: ACC, anterior cingulate cortex; alNS, anterior insula; ALFF, amplitude of low-frequency fluctuation; BG, basal ganglia; CPL, cerebellar posterior lobe; fO, frontal operculum; Fus, fusiform; IPL, inferior parietal lobule; ITG, inferior temporal gyrus; JOA, Japanese Orthopaedic Association; LBLP, low-back and leg pain; MB, midbrain; MOG, middle occipital gyrus; mPFC, medial prefrontal cortex; MTG, middle temporal gyrus; OFC, orbitofrontal cortex; PFC, prefrontal cortex; PoCG, postcentral gyrus; Thala, thalamus; TPTD, two-point tactile discrimination; VAS, visual analog scale.

\section{Publish your work in this journal}

The Journal of Pain Research is an international, peer reviewed, open access, online journal that welcomes laboratory and clinical findings in the fields of pain research and the prevention and management of pain. Original research, reviews, symposium reports, hypothesis formation and commentaries are all considered for publication.

\section{Dovepress}

The manuscript management system is completely online and includes a very quick and fair peer-review system, which is all easy to use. Visit http://www.dovepress.com/testimonials.php to read real quotes from published authors. 